\title{
BMJ Open Point-of-care viral load testing among adolescents and youth living with HIV in Haiti: a protocol for a randomised trial to evaluate implementation and effect
}

Lindsey K Reif (D) , ${ }^{1,2}$ Marie Elmase Belizaire, ${ }^{3}$ Grace Seo, ${ }^{1}$ Vanessa Rouzier, ${ }^{1,3}$ Patrice Severe, ${ }^{3}$ Joseph Marie Joseph, ${ }^{3}$ Bernadette Joseph, ${ }^{3}$ Sandra Apollon, ${ }^{3}$ Elaine J Abrams, ${ }^{2,4,5}$ Stephen M Arpadi, ${ }^{4,5,6}$ Batya Elul, ${ }^{2}$ Jean W Pape, ${ }^{1,3}$ Margaret L McNairy, ${ }^{7}$ Daniel W Fitzgerald, ${ }^{1}$ Louise Kuhn ${ }^{4,6}$

To cite: Reif LK, Belizaire ME, Seo G, et al. Point-of-care viral load testing among adolescents and youth living with HIV in Haiti: a protocol for a randomised trial to evaluate implementation and effect. BMJ Open 2020;10:e036147. doi:10.1136/ bmjopen-2019-036147

- Prepublication history for this paper is available online. To view these files, please visit the journal online (http://dx.doi org/10.1136/bmjopen-2019036147).

Received 03 December 2019 Revised 25 July 2020 Accepted 27 July 2020

Check for updates

(c) Author(s) (or their employer(s)) 2020. Re-use permitted under CC BY-NC. No commercial re-use. See rights and permissions. Published by BMJ.

For numbered affiliations see end of article.

Correspondence to

Lindsey K Reif;

lir2020@med.cornell.edu

\section{ABSTRACT}

Introduction Adolescents living with HIV have poor antiretroviral therapy (ART) adherence and viral suppression outcomes. Viral load (VL) monitoring could reinforce adherence but standard VL testing requires strong laboratory capacity often only available in large central laboratories. Thus, coordinated transport of samples and results between the clinic and laboratory is required, presenting opportunities for delayed or misplaced results. Newly available point-of-care (POC) $\mathrm{VL}$ testing systems return test results the same day and could simplify VL monitoring so that adolescents receive test results faster which could strengthen adherence counselling and improve ART adherence and viral suppression.

Methods and analysis This non-blinded randomised clinical trial is designed to evaluate the implementation and effectiveness of POC VL testing compared with standard laboratory-based VL testing among adolescents and youth living with HIV in Haiti. A total of 150 participants ages 10-24 who have been on ART for $>6$ months are randomised 1:1 to intervention or standard arms. Intervention arm participants receive a POC VL test (Cepheid Xpert HIV-1 Viral Load system) with sameday result and immediate ART adherence counselling. Standard care participants receive a laboratory-based VL test (Abbott m2000sp/m2000rt) with the result available 1 month later, at which time they receive ART adherence counselling. VL testing is repeated 6 months later for both arms. The primary objective is to describe the implementation of POC VL testing compared with standard laboratory-based VL testing. The secondary objective is to evaluate the effect of POC VL testing on VL suppression at 6 months and participant comprehension of the correlation between $V L$ and ART adherence.

Ethics and dissemination This study is approved by GHESKIO, Weill Cornell Medicine and Columbia University ethics committees. This trial will provide critical data to understand if and how POC VL testing may impact adolescent ART adherence and viral suppression. If effective, POC VL testing could routinely supplement
Strengths and limitations of this study

- This randomised controlled trial evaluating POC VL testing is conducted among adolescents and youth ages 10-24 living with HIV - a group that is in need of evidenced-based interventions to improve disproportionally poor health outcomes.

- This study evaluates both the implementation of POC VL testing and the effect on health outcomes. If shown to be effective, POC VL testing could be scaled up to supplement standard laboratory-based testing for use among adolescents and youth and other high-risk populations.

- The study is being conducted at a single site which could limit generalisability, but the study site, GHESKIO, is the largest HIV/AIDS clinic in the Caribbean and findings from this trial could inform the design and implementation of similar studies in other settings.

standard laboratory-based VL testing among high-risk populations living with HIV.

Trial registration number NCT03288246.

\section{INTRODUCTION}

Adolescents and youth living with HIV are a vulnerable and underserved population and have the poorest treatment outcomes of any age group. ${ }^{2} 2$ Since antiretroviral therapy (ART) availability has expanded, the number of HIV-related deaths has decreased by $32 \%$ in adults globally, but increased by $50 \%$ in adolescents. ${ }^{34}$ Adolescence is a period marked by physiological and psychological changes as young people navigate biological, emotional and sexual development. Experiences such as establishing identity and independence, managing perceived or experienced stigma 
and disclosing status to friends or sexual partners, can be especially challenging during this period. Without the coping mechanisms which come with emotional and social maturity, adolescents are at high risk for inadequate adherence to an ART regimen leading to drug resistance, disease progression and onward transmission of HIV. ${ }^{56}$

HIV-1 RNA viral load (VL) is the biomarker which most effectively measures the continued effect of ART. ${ }^{7} \mathrm{~A}$ suppressed VL, defined as HIV-1 RNA $<1000$ copies/mL by the $\mathrm{WHO}$, is an informative biomarker indicating that ART is appropriately treating the virus, most likely because adherence is adequate. ${ }^{8}$ Adequate adherence to achieve VL suppression is approximately $80 \%-90 \%$ and median time to VL suppression after establishing adequate ART adherence ranges from 1 to 3 months, depending on the ART regimen. ${ }^{910}$ The WHO recommends VL monitoring (ie, regular measurement of the quantity of HIV-1 RNA in plasma using standard quantitative clinical assays) to evaluate HIV treatment, assess treatment failure and ensure prompt switch to second-line therapy for those on a failing regimen. A high VL among a patient with perfect adherence may indicate potential drug resistance. ${ }^{11-13}$ Returning and discussing VL results with patients has been shown to be a tool to support viral resuppression since it provides an opportunity for providers to reinforce adherence. ${ }^{14-16}$ Since adolescents will require treatment for years longer than adults, poor adherence, treatment failure and drug resistance can have more serious consequences making VL monitoring particularly important for this population. ${ }^{1718}$

VL is measured by molecular tests that amplify HIV RNA by PCR. These assays require a high level of technical expertise and laboratory capacity and thus in low resource settings have generally been established in central laboratories $^{19}$. Thus VL monitoring also requires coordinated transportation of specimens and results between clinical sites (urban, periurban and rural) and centralised laboratories. Sending samples to a central laboratory risks significant delays, misplaced or lost samples and results, and multiple patient visits. ${ }^{20-22}$

A simplified system, the Cepheid Xpert HIV-1 Viral Load system, is a point-of-care (POC) VL assay that uses individual cartridges which perform an integrated extraction and quantitative real-time PCR for quantitative measures of HIV RNA ${ }^{23}$. The Cepheid Xpert HIV-1 VL system can be used at health facilities by minimally trained staff and results returned to the patient on the same day. ${ }^{24}{ }^{25}$ This system gives clinicians the opportunity to identify suboptimal ART adherence sooner than standard assays and thus provide real-time feedback to patients about their adherence. This may lead to stronger counselling, and allow for quicker clinical decisions about appropriate treatment and treatment failure. Since adolescents are at high risk for non-adherence and failing treatment, returning VL results faster to reinforce adherence could be an opportunity to improve health outcomes.

From a feasibility standpoint, POC VL testing does not rely on the availability of laboratories with high technical expertise. Moreover, even when adequate laboratories exist, POC VL testing reduces the logistical complexity of transporting specimens and results to and from a clinical site. POC VL testing also reduces the amount of time between testing and results. Since results are available the same day of testing, this reduces the likelihood of patient attrition prior to receiving the result. We hypothesise that the timely return of a concrete, quantitative VL result may enhance the impact of adherence counselling, and assist the clinician in formulating a suitable treatment plan including regimen changes. From a psychological standpoint, we hypothesise that providing a blood sample and receiving the VL result in the same visit could help adolescents conceptualise the connection between behaviour (ART adherence) and potential for negative health outcomes (high VL). As adolescents develop cognitively, they are still transitioning from concrete thinking to abstract thinking and they may lack the conceptual understanding of long-term or currently unseen consequences of not adhering to a medication regimen. ${ }^{26-28}$

While the theoretical benefits of POC testing appear promising, drawbacks in their field implementation have been highlighted in the literature. POC VL testing requires a constant power supply, proper assay maintenance and quality assurance, and supply chain capacity to stock, store, and safely dispose of cartridges ${ }^{29}$. Evaluating the feasibility of implementing a new POC VL testing protocol is needed.

We designed a randomised clinical trial (NCT03288246) to evaluate the implementation of POC VL testing and its effect on health outcomes and knowledge compared with standard of care (SOC), laboratory-based VL testing, among adolescents and youth living with HIV. The primary objective of the study is to describe the implementation of POC VL testing compared with standard laboratorybased VL testing. We hypothesise that POC VL testing paired with same-day patient education and counselling will result in a higher proportion of participants receiving their test result (fewer samples lost or results not accurately linked back to charts), and shorter time between testing and participant receipt of results. The secondary objective of the study is to evaluate the effect of POC VL testing on VL suppression and participant comprehension of the correlation between ART adherence and VL suppression. We hypothesise that providing same-day VL test results accompanied by adherence counselling the same day as testing could improve these outcomes.

\section{METHODS AND ANALYSIS \\ Study design}

This study is designed as an unblinded randomised clinical trial among adolescents and youth aged 10-24 years living with HIV who have been on ART for $\geq 6$ months. Adolescents are randomised 1:1 to one of two arms: (1) the POC arm or (2) the SOC arm. In the POC arm, adolescents have a POC VL test and receive adherence counselling informed by the VL result the same day as testing. In the 
A

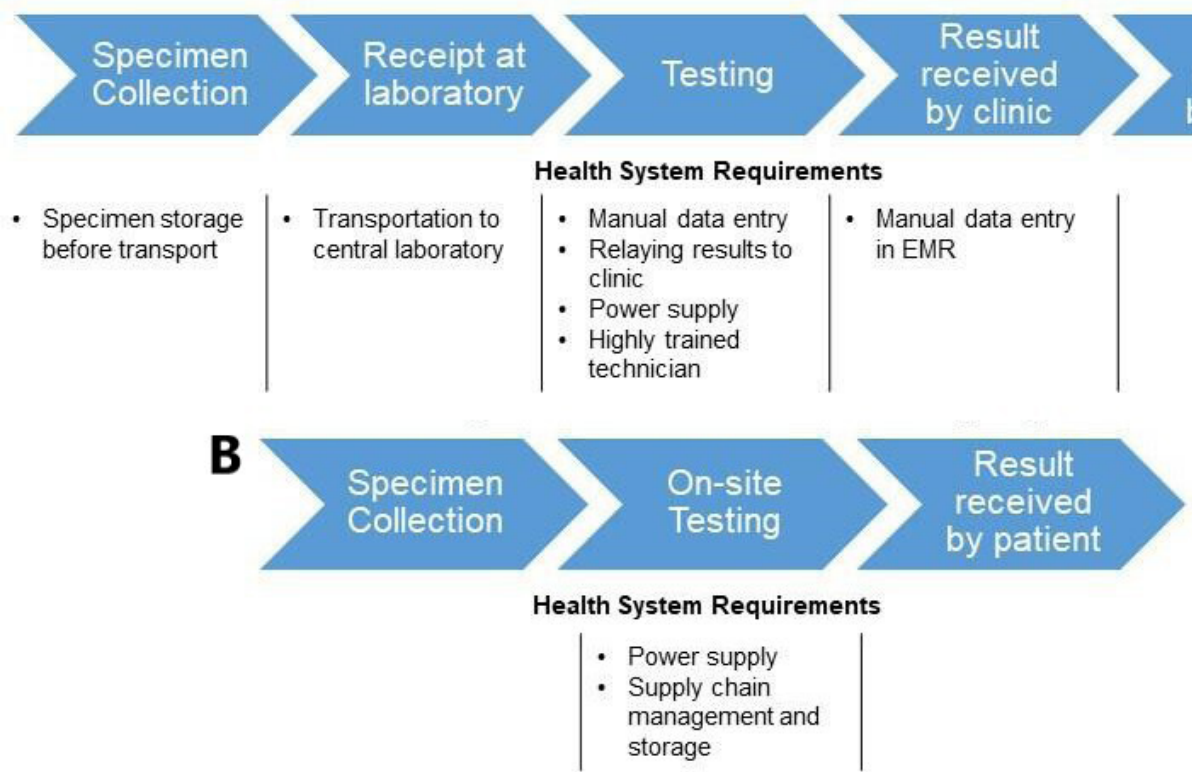

Figure 1 (A) Standard laboratory-based VL testing steps. (B) POC VL testing steps. EMR, electronic medical record; POC, point-of-care; VL, viral load.

SOC arm, adolescents have a standard laboratory-based test and then approximately 1 month later, once the VL test result is processed at the central laboratory and the result is linked back to the electronic medical record (EMR), they receive adherence counselling informed by the VL result at the follow-up clinic visit. The steps between sample collection and participant receipt of VL results, comparing the POC and SOC arms are described in figure $1 \mathrm{~A}, \mathrm{~B}$. A randomised design allows us to rigorously evaluate the implementation of POC VL testing compared with standard laboratory-based VL testing comparing the steps involved with processing the VL test in each system. The randomised design will also minimise potential bias due to confounding factors in evaluation of the impact of POC VL testing on VL suppression and comprehension of the correlation between VL and ART adherence.

\section{Study site}

The study takes place at GHESKIO, the French acronym for the Haitian Group for the Study of Kaposi's Sarcoma and Opportunistic Infections, an HIV/AIDS clinic in Port-au-Prince, Haiti. GHESKIO is the largest provider of HIV care in the Caribbean and provides HIV counselling and testing to nearly 40000 patients and ART to 20000 patients annually. GHESKIO has an on-site satellite laboratory which performs rapid tests for HIV and syphilis and urine pregnancy testing.

\section{Study intervention}

Intervention arm: POC VL testing

The intervention arm participants are asked to arrive for their visit before 11:00. They receive the Cepheid GeneXpert HIV-1 POC VL test. The blood sample is collected onsite at the phlebotomy clinic and processed onsite by a laboratory technician. Processing includes centrifuging the blood sample for $15 \mathrm{~min}$ to separate plasma from whole blood, and then the Cepheid GeneXpert HIV-1 VL system automates the testing processes including RNA extraction, purification, reverse transcriptions and cDNA real-time quantification in one cartridge within 90 min. We chose the Cepheid GeneXpert HIV-1 VL testing system because minimally trained, onsite staff can test samples individually and on-demand. ${ }^{25}{ }^{30}$ The assay is WHO prequalified for use in HIV management with plasma $^{31}$ and in an evaluation to determine its performance relative to the laboratory-based Abbott m2000sp/ m2000rt assay, with a similar threshold of detection (40 copies $/ \mu \mathrm{L}$ ), was highly concordant ${ }^{23}$.

The POC VL test result is processed and returned to the study nurse within 3 hours of collecting the blood sample, which the study nurse then provides to the participant with immediate adherence counselling. In the event that the test result is not available the same day (eg, there is an error with the POC VL assay or the participant arrives at the clinic too late for the test to be processed the same day), the clinician receives the result from the onsite laboratory the next day and the participant is called to return for the result. Participants are followed monthly and receive a follow-up POC VL test at month 6 .

\section{Control arm: standard laboratory-based VL testing}

In the current SOC, participants receive a standard laboratory-based VL test which requires samples to be sent offsite to a central laboratory for processing ( $\sim 1$ hour drive from the clinic). At the central laboratory, they are stored, batched with other samples and run weekly in the fully automated Abbott m2000sp/m2000rt system. Once the result is available, it is manually entered by laboratory 
staff into an Excel 2013 spreadsheet which is emailed to the clinic and then manually entered by data management staff into the EMR. At the next visit, if results are available, the clinician retrieves them from the EMR to return to the patient and provide appropriate adherence counselling informed by the result. The duration of this process-from sample collection to patient return of results-varies depending on the volume of samples collected each day and on laboratory and data management staffing. Average time from blood collection to availability of the result in the EMR is 2-3 weeks. Per standard clinical care, adolescents receive the VL result and adherence counselling informed by the result at their next visit, 1 month after providing the blood sample. There is no mechanism for 'flagging' a high VL to alert a clinician and subsequently the participant. We have made as little modification to this standard protocol as possible. Participants are followed monthly and SOC arm participants receive adherence counselling informed by the VL test result if it is available in the EMR at the next monthly visit.

Participants in both arms receive a reminder phone call 1 week before a visit; on this call the POC arm participants are reminded to arrive before 11:00 for a visit with a VL test scheduled. Participants in both arms receive the same adherence counselling curriculum and messages following WHO guidelines ${ }^{32}$. The content of the adherence counselling is guided by the VL test result. For example, if a participant's VL is $<1000$ copies $/ \mu \mathrm{L}$, counselling includes positive feedback for ongoing ART adherence and a discussion on how to prepare for or prevent any potential challenges to maintain this. If a participant's VL result is $\geq 1000$ copies $/ \mu \mathrm{L}$, counselling includes identifying current barriers to ART adherence and plans for how to address these in order to improve adherence and they are scheduled for a repeat VL test 3 months from the initial VL test. A key difference between arms is the timing of the adherence counselling-intervention arm participants receive this the same day as testing (ie, month 1 and month 6), and SOC arm participants receive this 1 month after testing (ie, month 2 and month 7).

\section{Study objectives, outcomes and measurements}

The primary study objective is to describe the implementation of VL testing across study arms, including the proportion of participants who receive a VL result and the timeliness of the result (table 1). Process steps include: (1) generating a valid VL test result, (2) returning the VL test results to the participant and (3) providing adherence counselling informed by the VL result. We will describe the proportion of steps achieved, the time between each step and document reasons for any delays or failures to reach a step, comparing study arms. The primary outcome will compare the proportion of participants in each arm who receive their VL test result within 6 weeks of VL testing. This allows for a short time buffer for SOC arm participants if appointments are not scheduled exactly 1 month from the previous visit for reasons related to school, work, or holidays.
Table 1 Study objectives, outcomes and measurements

Objective 1: describe the implementation of POC VL testing compared with standard laboratory-based testing

\begin{tabular}{ll}
\hline Outcome & Measurement \\
Description of VL testing & Proportion of participants with: \\
steps & $\begin{array}{l}\text { 1. a valid VL test result generated } \\
\text { 2. a VL test result returned } \\
\text { 3. adherence counselling } \\
\text { informed by the VL result } \\
\text { received }\end{array}$
\end{tabular}

Receipt of VL test result Receive VL test result within 6 weeks

Objective 2: measure the effect of POC VL testing on health outcomes

$\begin{array}{ll}\text { Outcome } & \text { Measurement } \\ \text { VL suppression } & <1000 \text { copies/ } \mu \mathrm{L} \\ \text { Comprehension of the } & >60 \% \text { of correct answers on the } \\ \text { correlation between ART } & \text { VL knowledge questionnaire } \\ \text { adherence and VL result } & \end{array}$

*Adapted from assessing general HIV/AIDS and CD4 knowledge. ART, antiretroviral therapy; POC, point-of-care; VL, viral load.

The secondary study objective is to evaluate the effect of POC VL testing on health outcomes and participant knowledge including: (1) VL suppression at 6 months and (2) comprehension of the correlation between VL and ART adherence (table 1). VL suppression at 6 months is defined as a HIV-1 RNA VL $<1000$ copies $/ \mu \mathrm{L}$ using results from the laboratory-based Abbott VL test for both arms. Demonstrated comprehension, by the adolescent, of the correlation between ART adherence and VL is assessed using a VL knowledge questionnaire which was adapted from studies that assessed general HIV/AIDS knowledge ${ }^{33-35}$ This questionnaire is similar to questions used to assess a cognitive behavioural programme-the 'Lifesteps Programme'- to improve drug adherence and HIV knowledge among adolescents and youth. ${ }^{28}{ }^{36}$ Comprehension is measured as a binary outcome-participants who answer $\geq 60 \%$ of questions correctly on the VL knowledge questionnaire are defined as having demonstrated comprehension.

ART adherence will also be measured at 1, 4 and 6 months by self-report and assessed using a modified version of the AIDS Clinical Trials Group questionnaire to evaluate adherence by 7 day recall, which has been shown to be more accurate for adolescents and youth than 30 day recall assessments. ${ }^{37-41}$ Self-reported adherence is measured as a binary variable: 'suboptimal' and 'optimal' based on the answer to the question: 'In the last 7 days, on how many days did you miss at least one dose of any of your ART medicines'? A response of 1 or more missed doses is categorised as 'suboptimal' and no missed doses is categorised as 'optimal'.

At each visit with a VL test, participants also provide a dried blood spot sample of whole blood. Samples from 
a subset of 50 participants (the first 25 enroled in each arm) will be analysed to measure the cumulative level of tenofovir-diphosphate (TDF-DP) drug level in the blood using liquid chromatography-tandem mass spectrometry $^{42}$. This will provide an additional marker of ART adherence. It will also allow us to assess the correlation between cumulative TDF-DP drug concentration, VL and self-reported adherence to determine if an unsuppressed $\mathrm{VL}$ is a result of an ineffective drug regimen or poor adherence.

\section{Study population: eligibility and enrolment}

All adolescents who had a VL test $\geq 6$ months prior are screened for invitation to the study. Specific inclusion criteria include adolescents and youth living with HIV aged 10-24 who have been on ART $\geq 6$ months as documented in the GHESKIO EMR; permanent residence in Port-au-Prince, and able to provide informed consent/ assent. Exclusion criteria include participants who are pregnant at enrolment; coinfected with tuberculosis or other comorbidities including cognitive impairment, bipolar disorder, psychosis; are in current need of inpatient psychiatric hospitalisation; or require an urgent VL test or an ART regimen change the day of enrolment. All participants are followed at GHESKIO for the duration of the study.

Eligible adolescents are referred to research staff who provide an overview of the study, evaluate participant understanding of the study and complete study enrolment for interested participants. Adolescents and youth 18 years and older provide written consent and adolescents younger than 18 years provide written assent with written parental or guardian consent.

\section{Study activities and data collection}

Participants are randomised (1:1) using a computer randomisation software to either the POC arm or the SOC arm at enrolment and followed monthly, per standard clinical protocol. Study-scheduled VL tests are conducted at month 1 and month 6. Participants with a VL $>1000$ copies/ $\mu \mathrm{L}$ at month 1 have a repeat VL test done at month 4 with adherence counselling. This adheres to standard clinical care-patients with a $\mathrm{VL}>1000$ copies $/ \mu \mathrm{L}$ receive counselling to improve adherence and are scheduled for a repeat VL test 3 months later (figure 2).

All participants complete a baseline questionnaire at study enrolment which captures sociodemographic data and clinical history. All participants complete the VL knowledge questionnaire at baseline and 1 month after receiving VL results and adherence counselling, to evaluate change in knowledge after receiving VL results and adherence counselling. All participants in the POC arm complete a questionnaire evaluating their experience with POC VL testing to determine if they would accept POC VL testing as standard care. All participants complete an adherence assessment at month 1,4 and 6. The timings of study activities including all questionnaires are listed in table 2. Data quality control and assurance are conducted
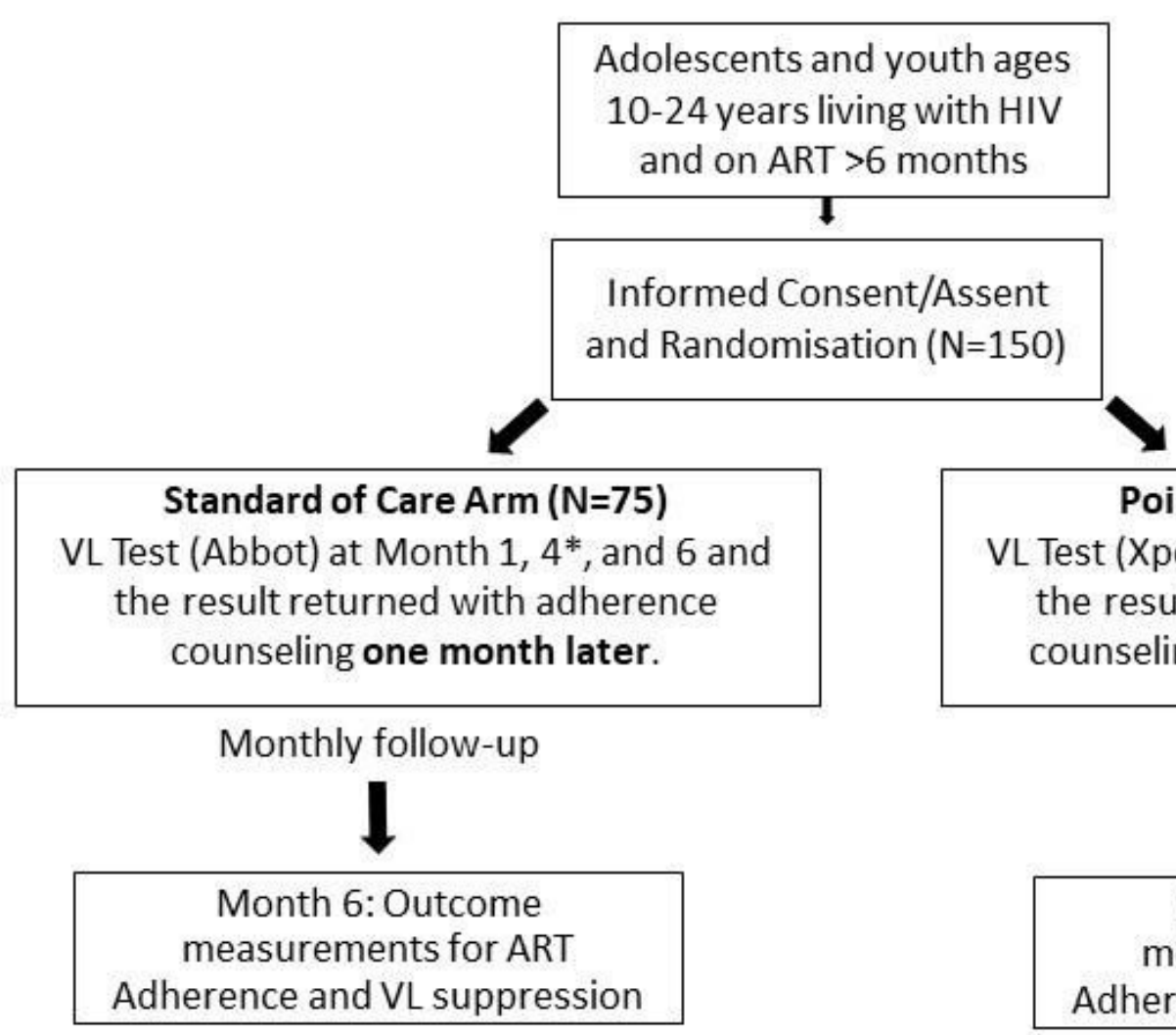

Point of Care Arm ( $\mathrm{N}=75)$

VL Test (Xpert) at Month 1, 4*, and 6 with the result returned with adherence counseling the same day as testing.

Monthly follow-up

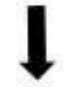

\begin{tabular}{c} 
Month 6: Outcome \\
measurements for ART \\
Adherence and VL suppression \\
\hline
\end{tabular}

Figure 2 Study schema. *Month 4 VL test administered only to those with a VL >1000 copies/ $/$ L at month 1 . ART, antiretroviral therapy; VL, viral load. 
Table 2 Schedule of study activities and follow-up

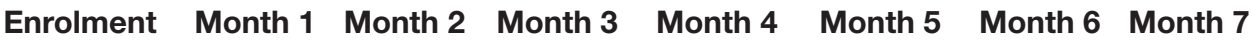

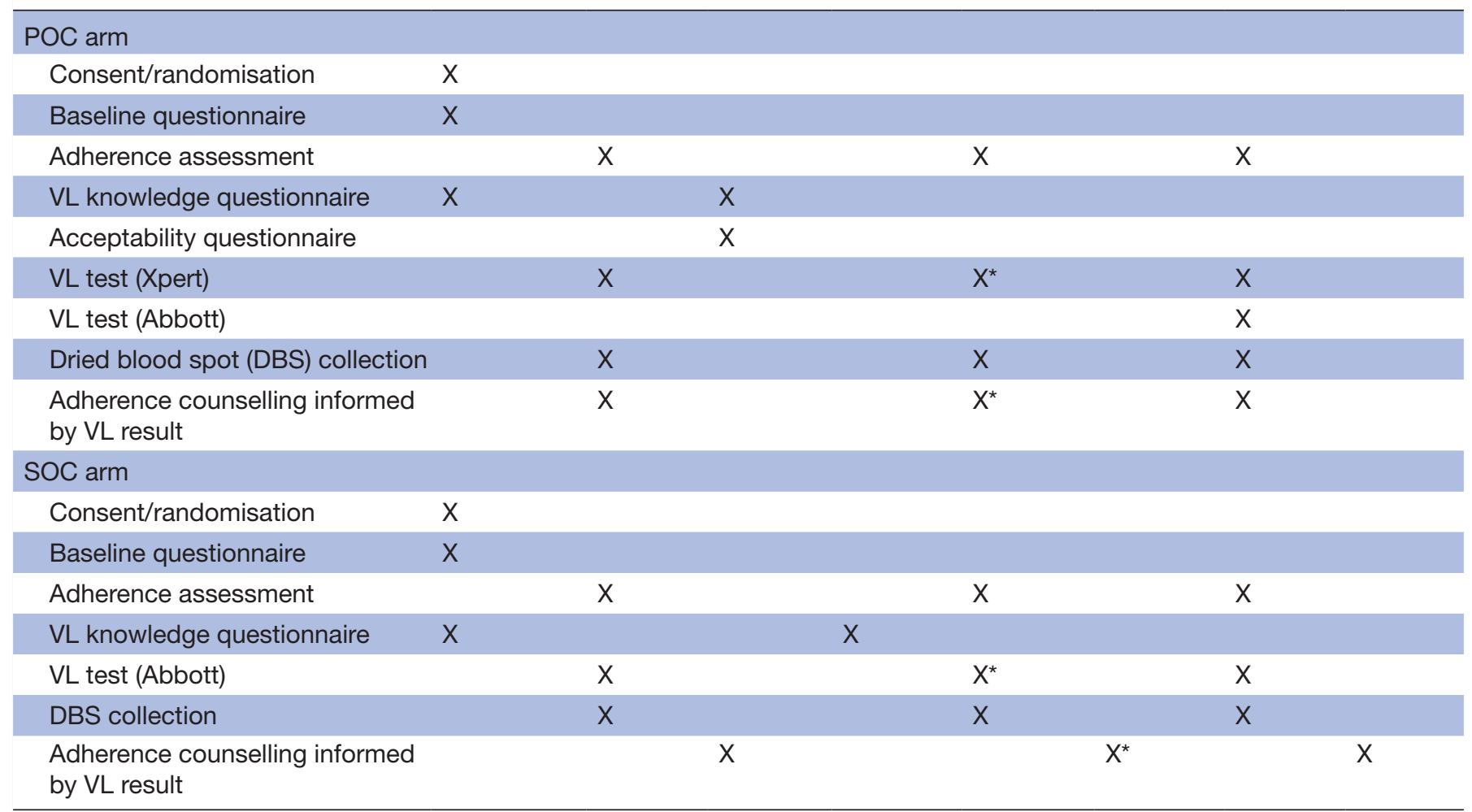

${ }^{*}$ Only if participant has $\mathrm{VL}>1000$ copies/ $\mu \mathrm{L}$ at month 1.

POC, point-of-care; SOC, standard of care; VL, viral load.

regularly for entry errors. Standard data collection, assessment and reporting for any adverse events are also conducted.

\section{Sample size and power calculations}

We calculated the required sample size to address the primary outcome of the study: the proportion of adolescents who receive a VL result within 6 weeks of the month 1 VL test. We calculated the sample size based on a retrospective review of VL results available in the EMR and interviews with providers at GHESKIO and hypothesise the primary outcome will be common. Between July 2016 and December 2016, we observed that 50\% of participants' VL results were entered into the EMR within 6 weeks of sample collection and confirmed this with providers in the clinic. The method we have chosen for calculation of the sample size is a simple comparison of proportions. We assume two-tailed tests, set a significance level at $5 \%$ and power of $80 \%$. For the primary outcome, with a sample size of 124 (62 per arm) we will have $80 \%$ power to detect a $20 \%$ increase (on the additive scale) in the proportion of patients who receive their VL result within 6 weeks of the month 1 VL test (ie, $70 \%$ in the SOC arm vs $90 \%$ in the POC arm).

We also calculated the required sample size to address a secondary outcome of the study: the proportion of adolescents who achieve a VL $<1000$ copies $/ \mu \mathrm{L}$ at 6 months. We anticipate that the secondary outcome will be slightly less common. A cross-sectional retrospective analysis of VL results among adolescents aged 10-24 who had been on ART $\geq 6$ months at the GHESKIO adolescent HIV clinic in 2016 showed $40 \%$ were virally suppressed. For the secondary outcome, with the same sample size of 124 (62 per arm), we will have $80 \%$ power to detect a $25 \%$ increase in the proportion of participants who are virally suppressed at 6 months from the index VL test (ie, $40 \%$ in the SOC arm vs $65 \%$ in the POC arm). Assuming we will achieve $85 \%$ follow-up in both arms, we are enroling a total sample size of 150 (75 per arm).

\section{Analysis and statistical methods}

We will conduct an 'intent to treat' analysis comparing participant outcomes by randomised study arm. We will compare the baseline characteristics between arms to determine if our randomisation process achieved a balance of these characteristics (eg, age, gender, presumed route of HIV transmission, age at ART initiation, time on ART, current ART regimen, baseline ART adherence and baseline VL). For the primary outcome, we will compare the proportion of participants who receive the month 1 VL test result within 6 weeks of testing, by randomisation arm. For the secondary outcomes, we will compare the proportion of participants who achieve or sustain a VL $<1000$ copies/ $\mu \mathrm{L} 6$ months from the month $1 \mathrm{VL}$ test, by randomisation arm. We will conduct secondary analyses to examine predictors of all outcomes (eg, time on ART, 
presumed route of HIV transmission) and assess whether there are subgroups among which these outcomes are more commonly achieved. For all outcomes, we will conduct statistical comparisons using $\chi^{2}$ tests for categorical variables, and t-tests for bell-shape distributed continuous variables. All $p$ values will be two-tailed. All analyses will be performed using $\mathrm{R}$ 3.6.3.

\section{Patient and public involvement}

The GHESKIO Community Advisory Board (CAB) facilitates communication between the GHESKIO clinic and Haitian communities. The $\mathrm{CAB}$ includes adolescent members who are specifically involved in discussions on projects involving adolescents and youth. The study was reviewed with the Adolescent $\mathrm{CAB}$ during one of their quarterly meetings and participants provided feedback on the design and implementation. At the end of the study, the $\mathrm{CAB}$ will comment on the findings and will contribute to the dissemination plan.

\section{DISCUSSION}

HIV-infected adolescents and youth are a particularly vulnerable population and central to the global HIV epidemic. Early and prolonged virologic suppression is associated with better clinical and neurocognitive outcomes among HIV-infected children and adolescents. $^{43} 44$ We hypothesise that POC VL testing, with same-day return of results, will lead to earlier identification of patients with poor adherence, earlier opportunities to intervene and ultimately earlier achievement of sustained ART adherence and viral suppression. If shown to be feasible and clinically effective, POC VL testing could be scaled up to routinely supplement standard laboratory-based VL testing for use among high-risk populations such as adolescents.

While the theoretical benefits of POC technologies appear substantial, drawbacks in their field implementation have been highlighted in the literature. ${ }^{2945-50}$ Simple 'scale-up' of POC testing has to take into consideration the larger health system and the subsequent changes that POC testing introduces. These changes include shifts in the supply chain which require clinics to monitor stock of different and potentially more complex supplies as well as securing a constant power supply and extra storage space. While designed to simplify processes, POC testing increases clinic responsibility and requires additional capacity. ${ }^{48}{ }^{49}$ POC testing also places additional responsibility on the provider and a longer visit is perceived negatively by those already overburdened with high patient volume. Finally, POC testing also changes the patient experience including longer visits. For example, one study has shown that some patients still choose to return for their test results at a later visit if POC testing lengthens the visit (eg, waiting 1 hour for result $)^{51}$. If POC technologies are not implemented with consideration for the larger health system and potential unintended consequences, poor uptake can limit their feasibility and impact. In this trial, we will evaluate the implementation of POC VL testing to determine if any of these potential drawbacks will impact this new protocol and to what extent.

Our trial has several limitations to note. We have a relatively small sample size and the study is being conducted at a single site, which could limit the generalisability of our results to other locations and among other populations. However, GHESKIO is the largest HIV/AIDS clinic in the Caribbean, and the findings of this trial could be used to design and implement similar studies to evaluate the impact of a POC VL testing intervention in other settings. Second, the POC VL testing intervention is narrow in scope when considering the magnitude of barriers that impede adequate ART adherence among adolescents. We recognise it is optimistic that returning a VL test result the same day can make widespread impact to improve ART adherence and viral suppression. If POC VL testing is shown to be effective, it could be used in combination with a package of additional interventions which address other patient-level barriers like stigma or lack of social support. We also acknowledge that there are potential unintended consequences to returning test results the same day-adolescents may feel 'caught' or shamed if the test result does not align with their reported selfadherence $^{52}$. However, counsellors are comprehensively trained to never convey blame or shame to the adolescent, but rather to show acceptance that adherence is difficult and assure participants they will provide intensive support to make improvements.

Adolescent and youth HIV outcomes remain far from the Joint United Nations Programme on HIV/AIDS (UNAIDS) 90-90-90 targets and innovative interventions are needed to both decrease morbidity and mortality among this vulnerable population and curb onward transmission of HIV. This trial will provide evidence as to whether a POC VL test is feasible and can simplify the VL testing process, and importantly whether it will result in faster clinical decision-making and improved health outcomes for adolescents with HIV. ${ }^{53} 54$

\section{Ethics and dissemination}

This study is approved by the GHESKIO, Weill Cornell Medicine and Columbia University ethics committees. All participants sign an informed consent form, and assent form as indicated, prior to any involvement in the study. The trial began enrollment in May 2018 and completed in August 2019. The study results will be presented at international scientific conference and will be submitted for publication in peer-reviewed journals.

\footnotetext{
Author affiliations

${ }^{1}$ Center for Global Health, Department of Medicine, Weill Cornell Medicine, New York, New York, USA

${ }^{2}$ Department of Epidemiology, Mailman School of Public Health, Columbia University Irving Medical Center, New York, New York, USA

${ }^{3}$ GHESKIO, Port-au-Prince, Ouest, Haiti

${ }^{4}$ ICAP at Columbia University, Mailman School of Public Health, Columbia University Irving Medical Center, New York, New York, USA
} 
${ }^{5}$ Department of Pediatrics, Vagelos College of Physicians and Surgeons, Columbia University Irving Medical Center, New York, New York, USA

${ }^{6}$ Gertrude H. Sergievsky Center, Vagelos College of Physicians and Surgeons, Columbia University Irving Medical Center, New York, New York, USA ${ }^{7}$ Division of General Internal Medicine, Department of Medicine, Weill Cornell Medicine, New York, New York, USA

Contributors LKR designed the study and is conducting data analysis. MEB, JM and $\mathrm{BJ}$ are conducting participant enrolment and follow-up. SA contributed to the study design and is conducting staff training and $Q A / Q C$ for all laboratory-related activities. GS is conducting data cleaning and assisting with analysis. VR and PS conducted study staff training and are overseeing study implementation including recruitment, enrolment and participant follow-up. EJA, SMA, BE, MLM, DWF, JWP and LK contributed to the design of the study. LK was also a major contributor to the writing of the manuscript. All authors read and approved the final manuscript.

Funding Three foundation grants supported the clinical services for participants enroled in this trial: MAC AIDS Foundation, Flora Family Foundation and Camela Basin Family Foundation. Two research training grants from the $\mathrm{NIH}$ supported training of study investigators: NIH/NIAID K24 Al098627 (PI: DWF) and NIH/FIC D43 TW010062 (PI: DWF).

\section{Competing interests None declared.}

Patient and public involvement Patients and/or the public were involved in the design, or conduct, or reporting or dissemination plans of this research. Refer to the Methods section for further details.

\section{Patient consent for publication Not required}

Provenance and peer review Not commissioned; externally peer reviewed.

Open access This is an open access article distributed in accordance with the Creative Commons Attribution Non Commercial (CC BY-NC 4.0) license, which permits others to distribute, remix, adapt, build upon this work non-commercially, and license their derivative works on different terms, provided the original work is properly cited, appropriate credit is given, any changes made indicated, and the use is non-commercial. See: http://creativecommons.org/licenses/by-nc/4.0/.

\section{ORCID iD}

Lindsey K Reif http://orcid.org/0000-0003-3427-4475

\section{REFERENCES}

1 Idele P, Gillespie A, Porth T, et al. Epidemiology of HIV and AIDS among adolescents: current status, inequities, and data gaps. $J$ Acquir Immune Defic Syndr 2014;66:S144-53.

2 Sohn AH, Hazra R. The changing epidemiology of the global paediatric HIV epidemic: keeping track of perinatally HIV-infected adolescents. J Int AIDS Soc 2013;16:18555.

3 UNICEF. Children and AIDS, statistical update. New York, 2015 Available: https://data.unicef.org/wp-content/uploads/2015/12/ 2015-Children-Adolescents-and-AIDS-Statistical-Update-ExecutiveSummary 244.pdf [Accessed 15 Aug 2019].

4 UNICEF. Towards an AIDS-free generation: children and AIDS sixth stock-taking report. New York, 2013. Available: https://data.unicef. org/resources/towards-an-aids-free-generation-children-and-aidsstocktaking-report-2013/ [Accessed 15 Aug 2019].

$5 \mathrm{Kim} \mathrm{MH}$, Mazenga AC, Yu X, et al. High self-reported nonadherence to antiretroviral therapy amongst adolescents living with HIV in Malawi: barriers and associated factors. J Int AIDS Soc 2017;20:1-12.

$6 \mathrm{Kim} \mathrm{S-H}$, Gerver SM, Fidler S, et al. Adherence to antiretroviral therapy in adolescents living with HIV: systematic review and metaanalysis. AIDS 2014;28:1945-56.

7 WHO. Antiretroviral therapy for HIV infection in adults and adolescents. recommendations for a public health approach. Geneva: World Health organization, 2010. Available: http:// whqlibdoc.who.int/publications/2010/9789241599764_eng.pdf [Accessed 15 Aug 2019].

8 Bennett DE, Bertagnolio S, Sutherland D, et al. The world Health organization's global strategy for prevention and assessment of HIV drug resistance. Antivir Ther 2008;13:1-13.

9 Byrd KK, Hou JG, Hazen R, et al. Antiretroviral adherence level necessary for HIV viral suppression using real-world data. J Acquir Immune Defic Syndr 2019;82:245-51.

10 Viswanathan S, Detels R, Mehta SH, et al. Level of adherence and HIV RNA suppression in the current era of highly active antiretroviral therapy (HAART). AIDS Behav 2015;19:601-11.
11 Bernheimer JM, Patten G, Makeleni T, et al. Paediatric HIV treatment failure: a silent epidemic. J Int AIDS Soc 2015;18:20090.

12 Pursuing Later Treatment Options II (PLATO II) project team for the Collaboration of Observational HIV Epidemiological Research Europe (COHERE), Castro H, Judd A, et al. Risk of triple-class virological failure in children with HIV: a retrospective cohort study. Lancet 2011;377:1580-7.

13 Jenabian MA, Costiniuk CT, Mboumba Bouassa RS. Chapdeleine Mekue Mouafo L, Brogan TV, Belec L. tackling virological failure in HIV-infected children living in Africa. Expert Rev Anti Infect Ther 2015;13:1213-23.

14 Bonner K, Mezochow A, Roberts T, et al. Viral load monitoring as a tool to reinforce adherence: a systematic review. J Acquir Immune Defic Syndr 2013;64:74-8.

15 Roberts T, Cohn J, Bonner K, et al. Scale-Up of routine viral load testing in resource-poor settings: current and future implementation challenges. Clin Infect Dis 2016;62:1043-8.

16 Ehrenkranz PD, Baptiste SL, Bygrave H, et al. The missed potential of CD4 and viral load testing to improve clinical outcomes for people living with HIV in lower-resource settings. PLoS Med 2019;16:e1002820.

17 Schwartz SR, Kavanagh MM, Sugarman J, et al. HIV viral load monitoring among key populations in low- and middle-income countries: challenges and opportunities. J Int AIDS Soc 2017;20.

18 Marcus R, Ferrand RA, Kranzer K, et al. The case for viral load testing in adolescents in resource-limited settings. J Int AIDS Soc 2017;20.

19 Harries AD, Zachariah R, van Oosterhout JJ, et al. Diagnosis and management of antiretroviral-therapy failure in resource-limited settings in sub-Saharan Africa: challenges and perspectives. Lancet Infect Dis 2010;10:60-5.

20 Calmy A, Ford N, Hirschel B, et al. HIV viral load monitoring in resource-limited regions: optional or necessary? Clin Infect Dis 2007;44:128-34

21 Ford N, Roberts T, Calmy A. Viral load monitoring in resourcelimited settings: a medical and public health priority. AIDS 2012;26:1719-20.

22 Nicholas S, Poulet E, Wolters L, et al. Point-Of-Care viral load monitoring: outcomes from a decentralized HIV programme in Malawi. J Int AIDS Soc 2019;22:e25387.

23 Moyo S, Mohammed T, Wirth KE, et al. Point-Of-Care Cepheid Xpert HIV-1 viral load test in rural African communities is feasible and reliable. J Clin Microbiol 2016;54:3050-5.

24 Ndlovu Z, Fajardo E, Mbofana E, et al. Multidisease testing for HIV and TB using the GeneXpert platform: a feasibility study in rural Zimbabwe. PLoS One 2018;13:e0193577.

25 Drain PK, Dorward J, Bender A, et al. Point-Of-Care HIV viral load testing: an essential tool for a sustainable global HIV/AIDS response. Clin Microbiol Rev 2019;32:1-3.

26 Choudhury S, Blakemore S-J, Charman T. Social cognitive development during adolescence. Soc Cogn Affect Neurosci 2006;1:165-74

27 Taddeo D, Egedy M, Frappier J-Y. Adherence to treatment in adolescents. Paediatr Child Health 2008:13:19-24.

28 Sriondee R. Effects of cognitive and behavioral program on drug adherence, CD4 and AIDS knowledge in late adolescent and early adult HIV/AIDS patients. Siriraj Med J 2016;67:14-19.

29 Pai NP, Wilkinson S, Deli-Houssein R, et al. Barriers to implementation of rapid and point-of-care tests for human immunodeficiency virus infection: findings from a systematic review (1996-2014). Point Care 2015;14:81-7.

30 Sacks JA, Fong Y, Gonzalez MP, et al. Performance of Cepheid Xpert HIV-1 viral load plasma assay to accurately detect treatment failure. AIDS 2019;33:1881-9.

31 WHO Prequalification of In Vitro Diagnostics. Public report. product: Xpert HIV-1 viral load with GeneXpert DX, GeneXpert Infinity-48, GeneXpert Infinity-48s and GeneXpert Infinity-80. July 2019, version 2.0. Available: https://wwwwhoint/diagnostics laboratory/ evaluations/pq-list/hiv- vrl/170720_final_pq_report pqdx_0192_0193_0194_0195_070-00pdf?ua=1 [Accessed 6 Jan 2020].

32 World Health Organization. HIV and adolescents: guidance for HIV testing, and counseling, and care for adolescents living with HIV. Geneva, Switzerland, 2013. Available: https://apps.who.int/iris/ bitstream/handle/10665/94334/9789241506168_eng.pdf; [Accessed 20 Nov 2019].

33 Jones D, Cook R, Rodriguez A, et al. Appointment adherence and HIV outcomes. AIDS Behav 2013;17:242-9.

34 Tique JA, Howard LM, Gaveta S, et al. Measuring health literacy among adults with HIV infection in Mozambique: development and validation of the HIV literacy test. AIDS Behav 2017;21:822-32. 
35 Ownby RL, Waldrop-Valverde D, Hardigan P, et al. Development and validation of a brief computer-administered HIV-related health literacy scale (HIV-HL). AIDS Behav 2013;17:710-8.

36 Safren SA, Otto MW, Worth JL. Life-steps: applying cognitive behavioral therapy to HIV medication adherence. Cognitive and Behavioral Practice 1999;6:332-41.

37 Chesney MA, Ickovics JR, Chambers DB, et al. Self-reported adherence to antiretroviral medications among participants in HIV clinical trials: the AACTG adherence instruments. Patient Care Committee \& Adherence Working Group of the Outcomes Committee of the Adult AIDS Clinical Trials Group (AACTG). AIDS Care 2000;12:255-66.

38 Reynolds NR, Sun J, Nagaraja HN, et al. Optimizing measurement of self-reported adherence with the ACTG adherence questionnaire: a cross-protocol analysis. J Acquir Immune Defic Syndr 2007;46:402-9.

39 Crane HM, Nance RM, Delaney JAC, et al. A comparison of adherence Timeframes using missed dose items and their associations with viral load in routine clinical care: is longer better? AIDS Behav 2017;21:470-80.

40 Stirratt MJ, Dunbar-Jacob J, Crane HM, et al. Self-Report measures of medication adherence behavior: recommendations on optimal use. Trans/ Behav Med 2015;5:470-82.

41 Intasan J, Bunupuradah T, Vonthanak S, et al. Comparison of adherence monitoring tools and correlation to virologic failure in a pediatric HIV clinical trial. AIDS Patient Care STDS 2014;28:296-302.

42 Castillo-Mancilla JR, Zheng J-H, Rower JE, et al. Tenofovir, emtricitabine, and tenofovir diphosphate in dried blood spots for determining recent and cumulative drug exposure. AIDS Res Hum Retroviruses 2013;29:384-90.

43 Crowell CS, Huo Y, Tassiopoulos K, et al. PACTG 219C study Team and the pediatric HIVAIDS cohort study (PHACS). early viral suppression improves neurocognitive outcomes in HIV-infected children. AIDS 2015;29:295-304.
44 Lazarus JR, Rutstein RM, Lowenthal ED. Treatment initiation factors and cognitive outcome in youth with perinatally acquired HIV infection. HIV Med 2015;16:355-61.

45 Schnippel K, Meyer-Rath G, Long L, et al. Scaling up Xpert MTB/RIF technology: the costs of laboratory- vs. clinic-based roll-out in South Africa. Trop Med Int Health 2012;17:1142-51.

46 Palamountain KM, Baker J, Cowan EP, et al. Perspectives on introduction and implementation of new point-of-care diagnostic tests. J Infect Dis 2012;205:S181-90.

47 Gous N, Scott L, Berrie L, et al. Options to expand HIV viral load testing in South Africa: evaluation of the GeneXpert ${ }^{\circ}$ HIV-1 viral load assay. PLoS One 2016;11:e0168244.

48 Pai NP, Pai M. Point-Of-Care diagnostics for HIV and tuberculosis: landscape, pipeline, and unmet needs. Discov Med 2012;13:35-45.

49 Pai NP, Vadnais C, Denkinger C, et al. Point-Of-Care testing for infectious diseases: diversity, complexity, and barriers in low- and middle-income countries. PLoS Med 2012;9:e1001306.

50 Meyer-Rath G, Schnippel K, Long L, et al. The impact and cost of scaling up GeneXpert MTB/RIF in South Africa. PLoS One 2012;7:e36966.

51 Mkwanazi NB, Patel D, Newell M-L, et al. Rapid testing may not improve uptake of HIV testing and same day results in a rural South African community: a cohort study of 12,000 women. PLoS One 2008;3:e3501.

52 Bernays S, Paparini S, Seeley J, et al. "Not Taking it Will Just be Like a Sin": Young People Living with HIV and the Stigmatization of LessThan-Perfect Adherence to Antiretroviral Therapy. Med Anthropol 2017;36:485-99.

53 Essajee S, Vojnov L, Penazzato M, et al. Reducing mortality in HIVinfected infants and achieving the 90-90-90 target through innovative diagnosis approaches. J Int AIDS Soc 2015;18:20299.

54 Gous N, Scott L, Potgieter J, et al. Feasibility of performing multiple point of care testing for HIV anti-retroviral treatment initiation and monitoring from multiple or single fingersticks. PLoS One 2013;8:e85265. 